\title{
Classification of Visual Perception and Imagery based EEG Signals Using Convolutional Neural Networks
}

\author{
Ji-Seon Bang \\ Dept. Brain and Cognitive Engineering \\ Korea University \\ Seoul, Republic of Korea \\ js bang@korea.ac.kr
}

\author{
Ji-Hoon Jeong \\ Dept. Brain and Cognitive Engineering \\ Korea University \\ Seoul, Republic of Korea \\ jh jeong@korea.ac.kr
}

\author{
Dong-Ok Won \\ Dept. Artificial Intelligence Convergence \\ Hallym University \\ Chuncheon, Republic of Korea \\ dongok.won@hallym.ac.kr
}

\begin{abstract}
Recently, visual perception (VP) and visual imagery (VI) paradigms are investigated in several brain-computer interface (BCI) studies. VP and VI are defined as a changing of brain signals when perceiving and memorizing visual information, respectively. These paradigms could be alternatives to the previous visual-based paradigms which have limitations such as fatigue and low information transfer rates. In this study, we analyzed VP and VI to investigate the possibility to control BCI. First, we conducted a time-frequency analysis with event-related spectral perturbation. In addition, two types of decoding accuracies were obtained with convolutional neural network to verify whether the brain signals can be distinguished from each class in the VP and whether they can be differentiated with VP and VI paradigms. As a result, the 6-class classification performance in VP was $32.56 \%( \pm 7.07)$ and the binary classification performance which classifies two paradigms was $\mathbf{9 0 . 1 6 \%}( \pm 9.69)$.
\end{abstract}

Keywords- visual imagery; visual perception; convolutional neural network

\section{INTRODUCTION}

Brain-computer interface (BCI) allows users to control external devices using their intentions, which are decoded from brain signals [1]-[4]. BCI involves several paradigms such as motor imagery (MI) [5], [6], event-related potential (ERP) [7], steady-state visually evoked potential (SSVEP) [8], [9], and rapid serial visual presentation (RSVP) [10], [11]. Also, there have been conducted several visual-based studies [12], [13]. Recently, visual imagery (VI) and visual perception (VP) are investigated as novel paradigms in several BCI studies [14], [15]. VP is defined as a changing of brain signal when

20xx IEEE. Personal use of this material is permitted. Permission from IEEE must be obtained for all other uses, in any current or future media, including reprinting/republishing this material for advertising or promotional purposes, creating new collective works, for resale or redistribution to servers or lists, or reuse of any copyrighted component of this work in other works.

This work was partly supported by Institute of Information \& Communications Technology Planning \& Evaluation (IITP) grant funded by the Korea government (MSIT) (No. 2015-0-00185, Development of Intelligent Pattern Recognition Softwares for Ambulatory Brain Computer Interface, No. 2017-0-00451, Development of BCI based Brain and Cognitive Computing Technology for Recognizing User's Intentions using Deep Learning, No. 20190-00079, Artificial Intelligence Graduate School Program(Korea University)). someone perceives certain visual information and VI is defined as a fluctuation of brain signal when someone memory certain visual information [16].

There have been several electroencephalography (EEG) studies based on VI and VP. Kosmyna et al. [14] analyzed what extent can the VI and VP be differentiated. They performed VP and VI while paying attention and imagining two images, a flower and a hammer. The following binary classification performances were presented: VP vs VI, VP of two classes, resting-state vs VP, and resting-state vs VI. In a study by Sousa et al. [15], VP and VI of 3-class were proposed. They provided static dot and moving dot as stimulation conditions. In specific, a static dot and a moving dot in the vertical axis, and a moving dot in vertical and horizontal axes are the 3-class that are used for the experiment. They conducted the time frequency analysis for both VP and VI using event-related spectral perturbation (ERSP) to represent the brain responses in the whole brain area. They found a decrease of alpha-band (8-13 Hz) power, especially in the area from the parietal to occipital during VP. Also, they found an increase of alpha-band power, especially in the area from the frontal to central during VI paradigm.

Besides, VP and VI-based magnetoencephalography study also exists. In a study by Dijkstra et al. [17], the author compared VP and VI to provide insights for the understanding of the neural mechanisms of visual processing. They showed that VI decoding becomes significant later compared to VP. This finding suggests that the entire visual representation is activated at once during VI. Also, they found out that whereas VP was characterized by high temporal specificity, VI showed low temporal specificity. A study by Spyropoulos et al. [13] represented the manifestation of visual tasks with electrocorticography. The author showed that a theta band $(3-8 \mathrm{~Hz})$ is predominant in visual attention tasks from the experiment with two macaque monkeys. They found this result by recording local field potential from the visual cortex, V1, V2, V4, and temporal-occipital (TEO) region.

Meanwhile, in the process of decoding the brain signal, 


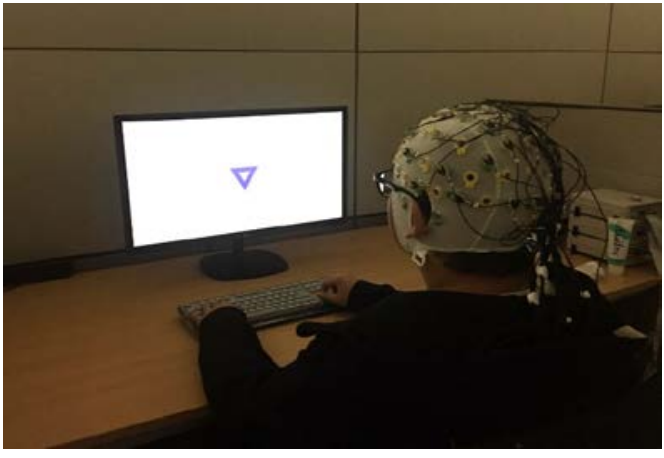

Fig. 1. The experimental environment of VP and VI paradigm.

the feature extraction methods are particularly important, as there exist large inter- and intra-subject variability in the EEG. Although classical feature extraction methods such as common spatial pattern (CSP) [18] and filter bank common spatial pattern (FBCSP) [19] are exist, the convolutional neural network (CNN) showed enhanced performance in the BCI researches recently [20]-[26]. For example, Cecotti et al. [20], first adopted CNN for decoding of P300, Stober et al. [21], classified music rhythm, and Manor et al. [22], classified RSVP with CNN. Sakhavi et al. [23], Schirrmeister et al. [24], and Bang et al. [25] adopted CNN in MI-based BCIs and Kwak et al. [26], adopted CNN in SSVEP based BCIs.

In this study, we investigate the possibility to control BCI. For this purpose, we conducted VP and VI experiments and analyzed them with 4 subjects. Six different colored shapes were provided for the visual stimuli. With the obtained brain signal, we conducted a time-frequency analysis. Also, from the temporal-spatial input feature of the signal, we obtained two types of decoding accuracy with CNN. First was a 6class classification in the VP paradigm to verify whether the brain signal can be differentiated with each class. The second was classification which classifies VP and VI paradigms to verify whether the brain signal can be differentiated with each paradigm.

\section{Materials AND Methods}

\section{A. Participants}

4 healthy participants (3 males and 1 female; aged 24 to 29) participated in the experiment. All participants were informed about the paradigm before the experiment. The experimental
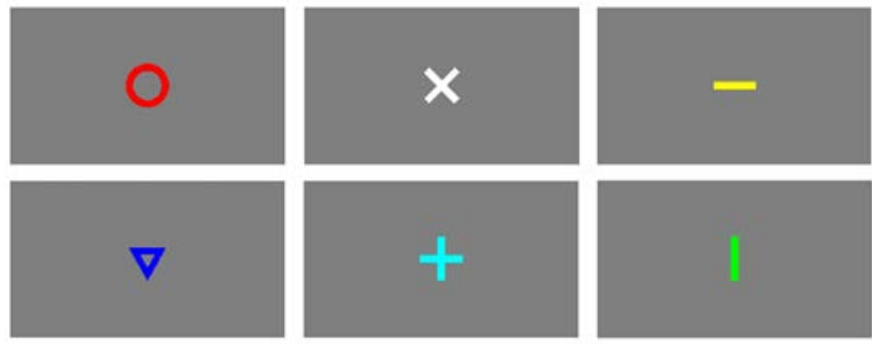

Fig. 2. Six visual stimuli which are provided for VP and VI.

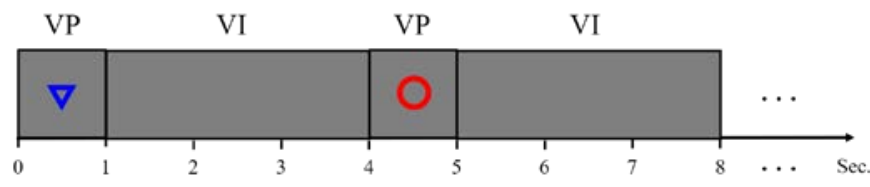

Fig. 3. The experimental paradigm.

protocols were reviewed and approved by the Institutional Review Board (IRB) at Korea University [KUIRB- 2020-005601].

\section{B. Visual Stimuli}

Figure 2 shows six stimuli used in this experiment. The six colored shapes were provided as visual stimuli for the experiment refer to Shen et al. [27]; red circle, white cross, yellow horizontal line, blue triangle, cyan plus, and green vertical line. The visual stimuli were presented at the center of the monitor. The size of the visual stimuli was $8 \mathrm{~cm} \times 8 \mathrm{~cm}$ and the distance between subject and monitor was $80 \mathrm{~cm}$ so that the degrees of viewing angle for the visual stimuli were $3^{\circ}$. This angle is matching with a foveal vision which refers to the center of the field of vision. When the image is focused in this area, the visual acuity is at its highest. By setting the viewing angle as $3^{\circ}$, the shape would be located to the foveal vision, so that the subject can recognize visual stimuli accurately without moving their eyes.

\section{Experimental Paradigm}

Figure 3 represents the experimental paradigm. For the VP experiment, subjects were instructed to pay attention to the shape which is appeared on the monitor for $1,000 \mathrm{~ms}$. After the shape disappears, the monitor becomes blank, and subjects were instructed to imagine the shape for 3,000 ms which was appeared before. This process is VI experiment. Six visual stimuli appear in random order in one cycle. When all six visual stimuli appear and disappear, one cycle is completed.

One cycle is defined as one trial and it lasts for $24 \mathrm{sec}$. The whole experiment is composed of 150 trials so that the total experiment time is one hour. To keep subjects pay attention, the experiment setup was designed to receive keyboard input from the subjects to move on to the next trial.

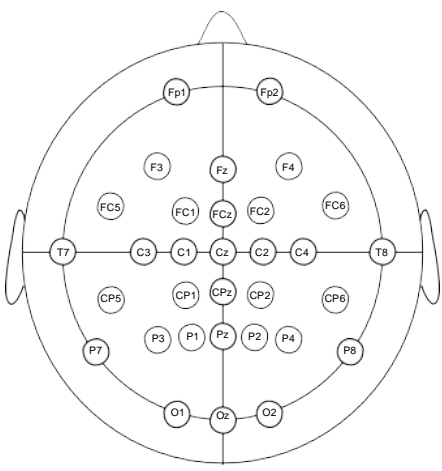

Fig. 4. The electrode channel montage which is used in the experiment. 

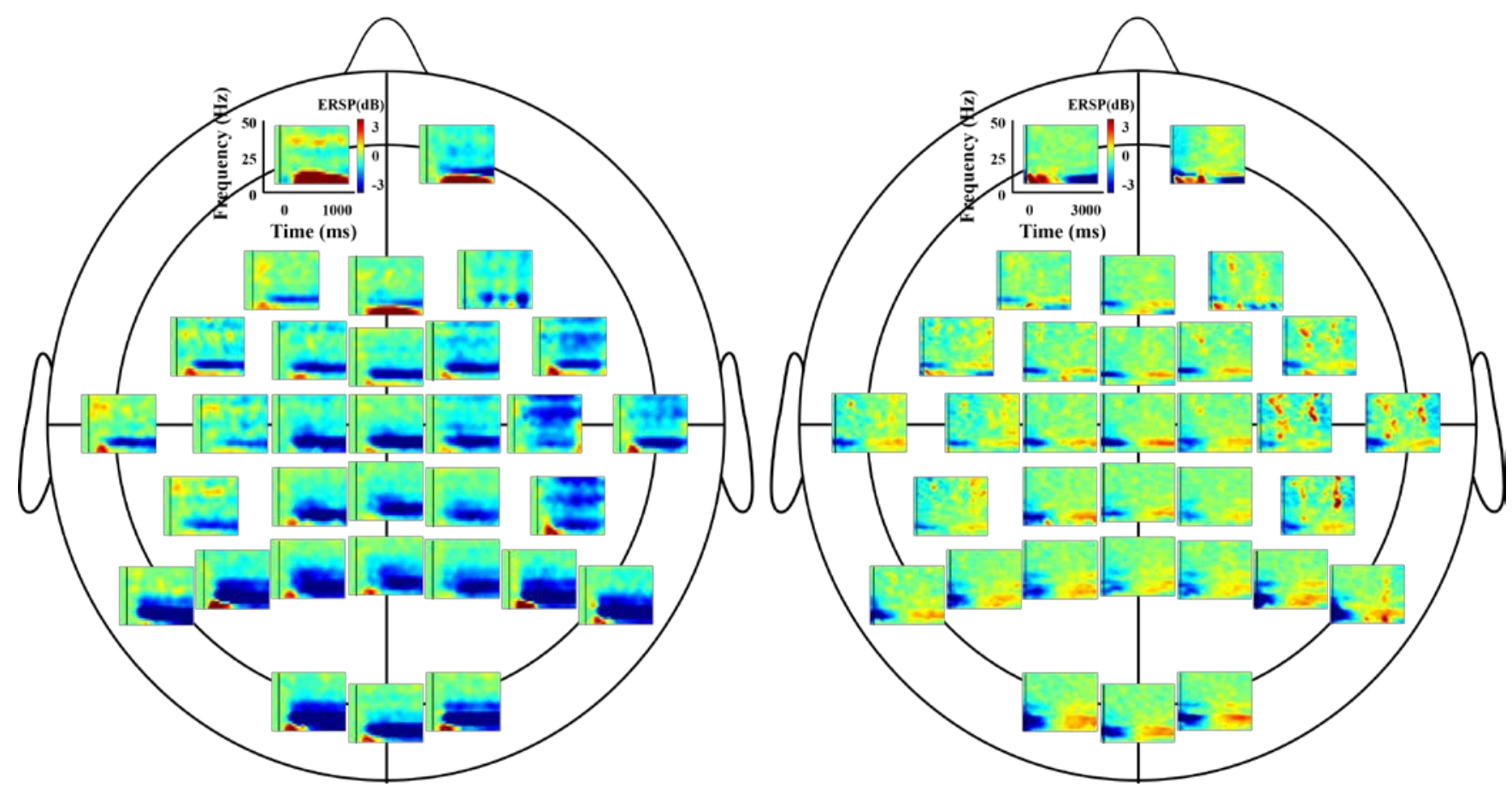

Fig. 5. Brain responses while conducting 1,000 ms VP (left) and 3,000 ms VI (right). The figure was represented based on ERSP.

\section{EEG Recording}

EEG data were recorded with $32 \mathrm{Ag} / \mathrm{AgCl}$ electrodes channels using a $1,000 \mathrm{~Hz}$ sampling rate according to the 10 20 international system (Fig. 4). EEG amplifier (BrainAmp, Brain Products) were used for the experiment. Reference and ground electrodes were fixed at posterior auricular and nasion, respectively.

\section{E. Time-Frequency Analysis}

We conducted a time-frequency analysis with ERSP with the OpenBMI toolbox (http://openbmi.org) [28]. The EEG data were band-pass filtered between 0.5 and $50 \mathrm{~Hz}$ for all channels, across entire trials. To analyze VP, the filtered data were segmented from -100 to $1,000 \mathrm{~ms}$, based on when the visual stimuli appeared. For VI, the signal from -100 to $3,000 \mathrm{~ms}$ was segmented based on when the visual stimuli disappeared. From each spectral estimate, the mean baseline log power spectrum was subtracted, to visualize power changes across the frequency range. This process produced baseline-normalized ERSP [29].

\section{F. Preprocessing}

We filtered and segmented both signals which were obtained while performing VP and VI for the classification. The signal from the VP was filtered with theta band (3-8 Hz) refer to [13] and was segmented from 0 to $1,000 \mathrm{~ms}$ based on the time the visual stimulus appeared. The signal obtained from the VI was filtered with the same band with VP and was segmented from 1,000 to $2,000 \mathrm{~ms}$ based on the time the visual stimulus was disappeared. The reason why we used not 0 but 1,000 for the start point was to remove VP effects.

To classify six classes in the VP paradigm, we used the preprocessed signal from VP. In addition, to classify each paradigm, we used both the preprocessed signal from VP and VI. With the segmented signal, we constructed temporalspatial input feature for CNN so that the size of the feature would be time points $(1,000) \times$ channels $(32)$.

\section{G. Data Classification with CNN}

The CNN network consists of 2 convolutional layers, 1 exponential linear unit (ELU) layer [30], 1 average-pooling layer, and 1 fully-connected layer. We designed the network so that each convolutional layer to be applied to the temporal features and the spatial features respectively. The first convolutional layer is adopted across the temporal domain, with 25 $\times 1$ sized filter. The second convolutional layer followed by ELU layer is adopted across spatial domain, with $1 \times 32$ sized filter. The filter size of average-pooling layer was $75 \times 1$ and the stride size was $15 \times 1$. This structure is the same for both 6-class classification in VP and binary classification which classifies VP and VI, except for the number of neurons in the classification layer (six and two, respectively). We employed Adam-optimizer [31] to optimize the cost. The learning rate was set to 0.001 and the iteration was set to 100 . We obtained 
Subject 1

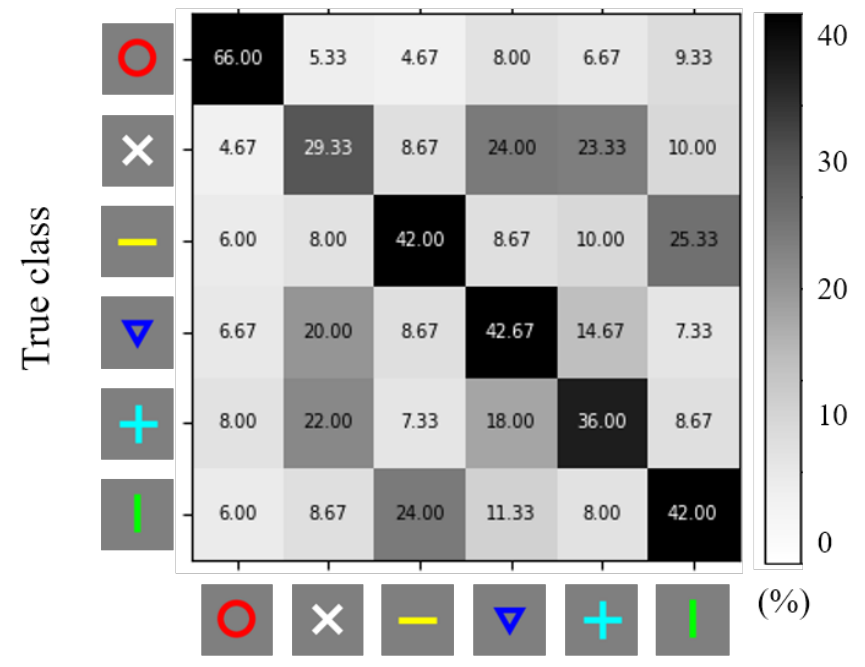

Predicted class
Subject 2

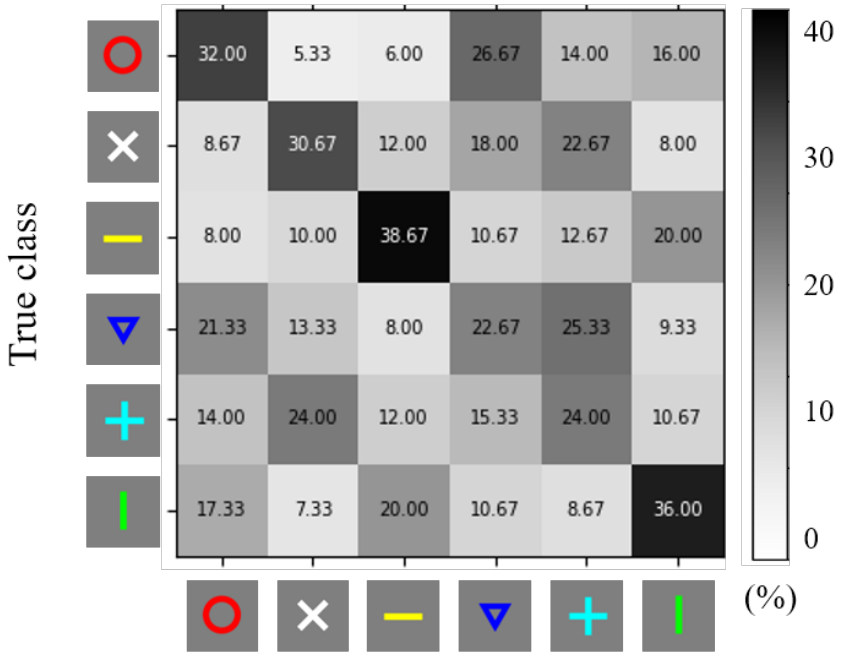

Predicted class

Fig. 6. The confusion matrix of VP for the subject 1 (left) and the subject 2 (right). Classification accuracy with 10-fold cross-validation is used.

the decoding accuracy by performing 10 -fold cross-validation for both classification results.

\section{RESULTS}

\section{A. ERSP Analysis}

We conducted a time-frequency analysis with ERSP. The analysis was conducted with all participants. The grand average of the brain responses while conducting VP (Figure 5 (left)) shows a significant decrease of theta and alpha-band power in the occipital area where the visual cortex is located. The analysis was also conducted for VI. The grand average of the brain responses while conducting VI (Figure 5 (right)) shows a decrease of alpha-band power until around 1,000 ms and the increase of alpha-band power after $1,000 \mathrm{~ms}$ in the whole-brain area.

\section{B. Classification Results}

In Figure 6, the confusion matrixes of 6-class visual perception for the subject 1 (left) and the subject 2 (right) are represented. Subject 1 and 2 are chosen as they achieved the highest and the next highest performance, respectively. The result was obtained based on 10-fold cross-validation.

TABLE I

Accuracy, Precision, Recall, and F1 Score From the Classification Result Between VP and VI

\begin{tabular}{c|c|c|c|c|c}
\hline & Sub1 & Sub2 & Sub3 & Sub4 & mean \\
\hline Accuracy & 0.988 & 0.961 & 0.772 & 0.884 & 0.902 \\
\hline Precision & 0.990 & 0.963 & 0.762 & 0.890 & 0.901 \\
\hline Recall & 0.987 & 0.958 & 0.790 & 0.878 & 0.903 \\
\hline F1 score & 0.988 & 0.960 & 0.776 & 0.884 & 0.902 \\
\hline
\end{tabular}

The figure shows that the subject 1 classified the first class (red circle) as the highest performance with $66.00 \%$, and the subject 2 classified the third class (yellow horizontal line) as the highest performance with $38.67 \%$. On the other hand, the subject 1 classified the second class (white cross) as the lowest performance with $29.33 \%$, and the subject 2 classified the fourth class (blue triangle) as the lowest performance with $22.67 \%$.

In Figure 7, the average performance of the 6-class classification in VP and the average performance of the binary classification which classifies VP and VI are presented. The chance level of two types of classification is also presented with the red line, which is $16.66 \%$ and $50 \%$, respectively. The results show that the decoding accuracies are $32.56 \%$ and $90.16 \%$, respectively.

In Table I, the classification result which classifies VP and VI are shown, based on the accuracy, precision, recall, and F1 score. The definition of each index is as follows:

$$
\begin{array}{r}
\text { Accuracy }=\frac{(T P+T N)}{(T P+F N+F P+T N)} \\
\text { Precision }=\frac{T P}{(T P+F P)} \\
\text { Recall }=\frac{T P}{(T P+F N)} \\
F 1 \text { score }=2 \times \frac{(\text { Precision } \times \text { Recall })}{(\text { Precision }+ \text { Recall })}
\end{array}
$$

where TP denotes true positive rate, TN denotes true negative rate, FN denotes false negative rate, and FP denotes false positive rate. 


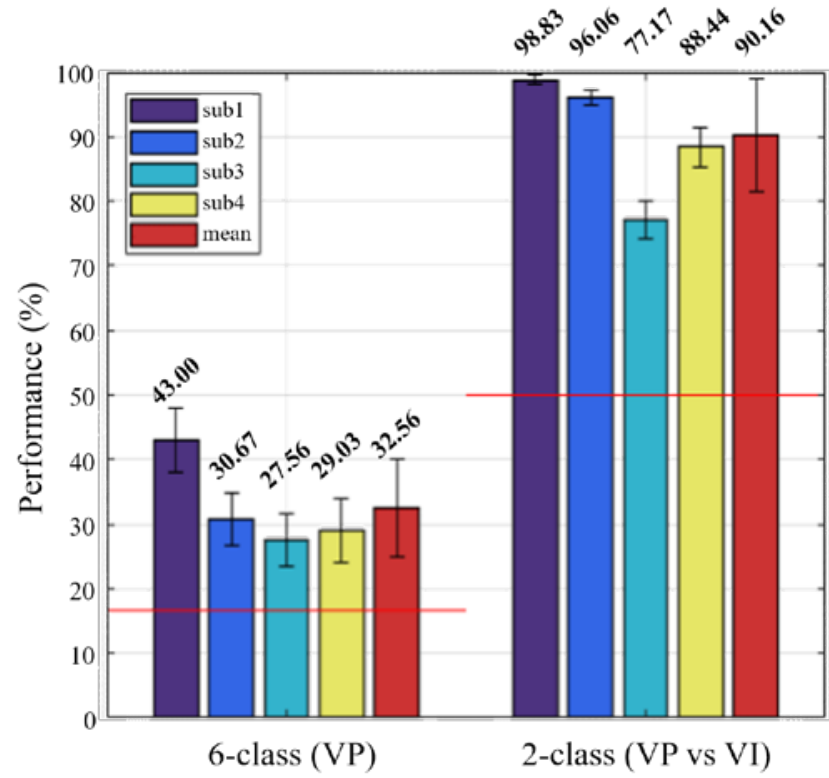

Fig. 7. The binary classification performance which classifies VP and VI (right) and 6-class classification performance in VP (left).

The result was obtained with 10 -fold cross-validation. In the result, subject 1 achieved the highest values for each index with $0.988,0.990,0.987$, and 0.988 , respectively. Subject 3 showed the lowest values for each index with $0.772,0.762$, 0.790 , and 0.776 , respectively.

\section{DISCUSSION}

In Figure 5 (left), the brain responses of the VP are represented. From the result, we found a significant decrease in theta and alpha-band power in the occipital area. These patterns are similar to the results which are represented from the previous studies [15]. It is well known that visual stimuli induce a decrease in the alpha-band power in the occipital area. This result indicates that the brain signal while performing VP is related to the perception of visual stimuli.

In Figure 5 (right), the brain responses of the VI are represented. From the result, we found that the decrease of alpha-band power followed by the increase of alpha-band power. The increase of alpha-band power is similar to that from the previous studies [15]. Whereas, for the decrease of alpha-band power which is appeared at the beginning, it appears that the signal generated by the VP is affected. This is because the VI experiment is right behind the VP experiment.

To avoid interference from VP, updating the experimental paradigm would be needed for the further improvement. To ensure that the VI paradigm does not come directly behind the VP paradigm, putting a random noise image between VP and VI can be one solution. We can expect the random noise image would minimize the impact of VP so that it will improve the quality of brain signals while performing VI.

In Figure 6, meanwhile, there is a difference between classes that are well classified and not well classified depending on each subject. The results show that the classes with the highest performance and the lowest performance were different for each subject. This indicates that to achieve stable performance, a feature extraction model should extract subject-dependent feature representation related to the visual character. Thus, further research should be to find subject-dependent visual features for each subject.

\section{CONCLUSION}

In this study, we conducted experiments of VP and VI with six different colored plane figures. From the experimental output, we analyzed brain responses during VP and VI. From the result, we found a significant decrease of theta and alphaband power while conducting VP and found that the increase of alpha-band power follows the decrease of alpha-band power while conducting VI. These results are similar to the results from previous work so that we could conclude that the brain signal from both paradigms is related to the perception and imagining of visual stimuli, respectively. Also, we obtained the decoding accuracy of 6-class classification in the VP paradigm, and 2-class classification which classifies VP and VI paradigms with deep learning methods. The results were $32.56 \%$ and $90.16 \%$, respectively. These results indicate that each class of VP can be classified and the brain signal while conducting VP and VI can be also differentiated as they showed significant performance, especially for the binary classification. From the result, we can conclude that the use of the proposed method is beneficial for the classification and prediction of the brain states from visual tasks (i.e. VP and VI) when developing comfortable BCI systems. Further study would be to control BCI systems through VP and VI or analyzing them in other paradigms such as magnetic resonance imaging (MRI) [32], positron emission tomography (PET) [33], or multi-modal paradigm [34].

\section{REFERENCES}

[1] G. Pfurtscheller and C. Neuper, "Motor imagery and direct brain computer communication,” Proc. IEEE, vol. 89, no. 7, pp. 1123-1134, Jul. 2001.

[2] J.-H. Kim, F. Bießmann, and S.-W. Lee, "Decoding three-dimensional trajectory of executed and imagined arm movements from electroencephalogram signals,” IEEE Trans. Neural. Syst. Rehabil. Eng., vol. 23, no. 5, pp. 867-876, Sep. 2015.

[3] I.-H. Kim, J.-W. Kim, S. Haufe, and S.-W. Lee, "Detection of braking intention in diverse situations during simulated driving based on EEG feature combination,” J. Neural. Eng., vol. 12, no. 1, pp. 016001, Nov. 2014.

[4] M. Lee, R.D. Sanders, S.-K. Yeom, D.-O. Won, K.-S. Seo, H.-J. Kim, G. Tononi, and S.-W. Lee, "Network properties in transitions of consciousness during propofol-induced sedation,” Sci. Rep., vol. 7, no. 1, pp. 1-13, Dec. 2017.

[5] H.-I. Suk and S.-W. Lee, "Subject and class specific frequency bands selection for multiclass motor imagery classification,” Int. J. Imaging. Syst. Technol., vol. 21, no. 2, pp. 123-130, Jun. 2011.

[6] T.-E. Kam, H.-I. Suk, and S.-W. Lee, "Non-homogeneous spatial filter optimization for electroencephalogram (EEG)-based motor imagery classification,” Neurocomputing, vol. 108, pp. 58-68, Feb. 2013.

[7] S.-K. Yeom, S. Fazli, K.-R. Müller, and S.-W. Lee, “An efficient ERPbased brain-computer interface using random set presentation and face familiarity," PLoS One, vol. 9, no. 11, pp. e111157, Nov. 2014.

[8] D.-O. Won, H.-J. Hwang, S. Dähne, K.-R. Müller, and S.-W. Lee, "Effect of higher frequency on the classification of steady-state visual evoked potentials,” J. Neural. Eng., vol. 13, no. 1, pp. 016014, Dec. 2015. 
[9] M.-H. Lee, O.-Y. Kwon, Y.-J. Kim, H.-K. Kim, Y.-E. Lee, J. Williamson, S. Fazli, and S.-W. Lee, "EEG dataset and OpenBMI toolbox for three BCI paradigms: An investigation into BCI illiteracy,” GigaScience, vol. 8, no. 5, pp. giz002, Jan. 2019.

[10] D.-O. Won, H.-J. Hwang, D.-M. Kim, K.-R. Müller, and S.-W. Lee, "Motion-based rapid serial visual presentation for gaze-independent brain-computer interfaces,” IEEE Trans. Neural Syst. Rehabil. Eng., vol. 26, no. 2, pp. 334-343, Feb. 2018.

[11] Y. Chen, A. D. Atnafu, I. Schlattner, W. T. Weldtsadik, M.-C. Roh, H.-J. Kim, S.-W. Lee, B. Blankertz, and S. Fazli, "A high-security EEG-based login system with RSVP stimuli and dry electrodes," IEEE Trans. Inf. Forensics. Secur., vol. 11, no. 12, pp. 2635-2647, Jun. 2016.

[12] H. H. Bülthoff, S.-W. Lee, T. A. Poggio, and C. Wallraven, Biologically Motivated Computer Vision. NY: Springer-Verlag, 2003.

[13] G. Spyropoulos, C. Bosman, and P. Fries, "A theta rhythm in macaque visual cortex and its attentional modulation,” Proc. Natl. Acad. Sci. U.S.A., vol. 115, no. 24, pp. E5614-E5623, Jun. 2018.

[14] N. Kosmyna, J. Lindgren, and A. Lecuyer, "Attending to visual stimuli versus performing visual imagery as a control strategy for EEG-based brain-computer interfaces,” Sci. Rep., vol. 8, no. 1, pp. 13222-13235, Sep. 2018.

[15] T. Sousa, C. Amaral, J. Andrade, G. Pires, U. Nunes, and M. CasteloBranco, "Pure visual imagery as a potential approach to achieve three classes of control for implementation of BCI in non-motor disorders," J. Neural. Eng., vol. 14, no. 4, pp. 1-11, Aug. 2017.

[16] M. Knauff, J. Kassubek, T. Mulack, and M. W. Greenlee, "Cortical activation evoked by visual mental imagery as measured by fMRI," Neuroreport, vol. 11, no. 18, pp. 3957-3962, Dec. 2000.

[17] N. Dijkstra, P. Mostert, F. Lange, S. Bosch, and M. Gerven, "Differential temporal dynamics during visual imagery and perception,” eLife, vol. 7, pp. e33904, May 2018.

[18] H. Ramoser, J. Muller-Gerking, and G. Pfurtscheller, “Optimal spatial filtering of single trial EEG during imagined hand movement," IEEE Trans. Rehabil. Eng., vol. 8, no. 4, pp. 441-446, Dec. 2000.

[19] K. K. Ang, Z. Y. Chin, C. Wang, C. Guan, and H. Zhang, "Filter bank common spatial pattern algorithm on BCI competition IV datasets 2a and 2b,” Front. Neurosci., vol. 6, pp. 39, Mar. 2012

[20] H. Cecotti and A. Graser, "Convolutional neural networks for P300 detection with application to brain-computer interfaces,” IEEE Trans. Pattern. Anal. Mach. Intell., vol. 33, no. 3, pp. 433-445, Mar. 2011.

[21] S. Stober, D. J. Cameron, and J. A. Grahn, “Using convolutional neural networks to recognize rhythm stimuli from electroencephalography recordings," in Adv. Neural. Inf. Process. Syst. (NIPS), Montreal, Canada, Jan. 2014, pp. 1449-1457.

[22] R. Manor and A Geva, “Convolutional neural network for multi-category rapid serial visual presentation BCI,” Front. Comput. Neurosci., vol. 9, pp. 146, Dec. 2015.

[23] S. Sakhavi, C. Guan, and S. Yan, "Learning temporal information for brain-computer interface using convolutional neural networks," IEEE Trans. Neural. Netw. Learn, Syst., vol. 29, no. 11, pp. 5619-5629, Mar. 2018.

[24] R. T. Schirrmeister, J. T. Springenberg, L. D. J. Fiederer, M. Glasstetter, K. Eggensperger, M. Tangermann, F. Hutter, W. Burgard, and T. Ball, "Deep learning with convolutional neural networks for EEG decoding and visualization,” Hum. Brain. Mapp., vol. 38, no. 11, pp. 5391-5420, Nov. 2017.

[25] J.-S. Bang, M.-H. Lee, S. Fazli, C. Guan, and S.-W. Lee, "Spatiospectral feature representation for motor imagery classification using convolutional neural networks,” IEEE Trans. Neural Netw. Learn. Syst., Jan. 2021.

[26] N.-S. Kwak, K.-R. Müller, and S.-W. Lee, “A convolutional neural network for steady state visual evoked potential classification under ambulatory environment," PLoS One, vol. 12, no. 2, pp. e0172578, Feb. 2017.

[27] G. Shen, T. Horikawa, K. Majima, and Y. Kamitani, "Deep image reconstruction from human brain activity,” PLoS Comput. Biol., vol. 15, no. 1, pp. e1006633, Jan. 2019.

[28] M.-H. Lee, K.-T. Kim, Y.-J. Kee, J.-H. Jeong, S.-M. Kim, S. Fazli, and S.-W. Lee, "OpenBMI: A real-time data analysis toolbox for brainmachine interfaces,” in Conf. Proc. IEEE Int. Conf. Syst. Man. Cybern. (SMC), Budapest, Hungary, Oct. 2016, pp. 001884-001887.

[29] A. Delorme and S. Makeig, "EEGLAB: An open source toolbox for analysis of single-trial EEG dynamics including independent component analysis,” J. Neurosci. Methods, vol. 134, no. 1, pp. 9-21, Mar. 2004.
[30] D. Clevert an T. Unterthiner and S. Hochreiter, "Fast and accurate deep network learning by exponential linear units (ELUs)," arXiv preprint arXiv:1511.07289, pp. 1-14, Nov. 2015.

[31] D. P. Kingma and J. Ba, “Adam: A method for stochastic optimization," arXiv preprint arXiv:1412.6980, pp. 1-15, Dec. 2014.

[32] X. Ding and S.-W. Lee, "Changes of functional and effective connectivity in smoking replenishment on deprived heavy smokers: A resting-state fMRI study,” PLoS One, vol. 8, no. 3, pp. e59331, Mar. 2013.

[33] X. Zhu an H.-I. Suk an S.-W. Lee and D. Shen, "Canonical feature selection for joint regression and multi-class identification in Alzheimer's disease diagnosis,” Brain. Imaging. Behav., vol. 10, no. 3, pp. 818-828, Sep. 2016.

[34] M.-H. Lee, S. Fazli, J. Mehnert, and S.-W. Lee, "Subject-dependent classification for robust idle state detection using multi-modal neuroimaging and data-fusion techniques in BCI,” Pattern. Recognit., vol. 48, no. 8, pp. 2725-2737, Aug. 2015. 